\title{
Paracrine induction of endothelium by tumor exosomes
}

\author{
Joshua L. Hood, Hua Pan, Gregory M. Lanza and Samuel A. Wickline, Consortium for Translational Research in \\ Advanced Imaging and Nanomedicine (C-TRAIN)
}

Cancers use a nanoscale messenger system known as exosomes to communicate with surrounding tissues and immune cells. However, the functional relationship between tumor exosomes, endothelial signaling, angiogenesis, and metastasis is poorly understood. Herein, we describe a standardized approach for defining the angiogenic potential of isolated exosomes. We created a powerful technique to rapidly and efficiently isolate and track exosomes for study using dynamic light scattering in conjunction with fluorescent exosome labeling. With these methods, melanoma exosomes were observed to interact with and influence endothelial tubule morphology as well as move between endothelial tubule cells by means of tunneling nanotube structures. Melanoma exosomes also were observed to rapidly stimulate the production of endothelial spheroids and endothelial sprouts in a dose-dependent manner. In concert, tumor exosomes simultaneously elicited paracrine endothelial signaling by regulation of certain inflammatory cytokines. These data suggest that, tumor exosomes can promote endothelial angiogenic responses, which could contribute to tumor metastatic potential. Laboratory Investigation (2009) 89, 1317-1328; doi:10.1038/labinvest.2009.94; published online 28 September 2009

KEYWORDS: exosomes; angiogenesis; tumor; cancer; endothelial; 3D assay

Cancer cells manipulate their microenvironment to optimize conditions for growth and metastasis in multiple ways. Recent evidence has suggested a novel mechanism for achieving this goal through the production and secretion of nanoscale membrane fragments known as tumor exosomes. ${ }^{1}$ Exosomes are naturally occurring biological nanoparticles $30-100 \mathrm{~nm}$ in size. $^{2-4}$ They are formed by the inward budding of multivesicular bodies (MVBs), a component of the endocytic pathway. Exosomes are constitutively generated and released into the surrounding extracellular matrix and circulation through fusion of MVBs with the plasma membrane. The nanoscale size of exosomes facilitates their penetration and interaction with host sites and cell types that are distant from an advancing tumor cell front and they have been shown to participate in cell to cell communication such as morphogen and RNA transport between cells. ${ }^{3}$ These transport processes can influence invasion of tumor cells, stimulate antigenic T-cell responses, modulate cell polarity and have a role in the developmental patterning of tissues. ${ }^{3}$ Exosomes may also have an important role in tumor immune evasion by direct suppression of immune cell activation. ${ }^{5-7}$
To metastasize to distant sites, tumor cells typically require a 'prepared environment' to establish successful implantation and growth. ${ }^{8}$ One model suggests that metastatic cells cooperate in waves. ${ }^{8}$ Early waves of metastatic cells prepare distal sites for interactions with subsequent waves to form metastatic foci. In addition, reactive lymph nodes that are downstream of tumors undergo lymphangiogenesis in preparation for tumor metastasis. ${ }^{9}$ Knowledge of how such sites are induced to become receptive to tumor implantation remains incomplete; although, in the case of melanoma, upstream secretion of soluble angiogenic signaling molecules such as VEGF have a role. ${ }^{9,10}$ Regardless of the mechanism, it would seem necessary that cancers, such as melanoma, would be capable of signaling site preparation for eventual metastasis. Moreover, little is known about the role of melanoma exosomes in angiogenesis. We hypothesize that melanoma exosomes might serve as nanocarriers of paracrine effectors of endothelium to prepare distal sites for docking of metastatic cells.

To elucidate the role of melanoma exosomes as nanovehicles that might pave the way for tumor cell implantation, 
we developed and validated a new in vitro three-dimensional (3D) angiogenesis assay system that incorporates a continuum of both tubulogenesis (early endothelial angiogenic changes) and spheroid sprouting (later endothelial angiogenic changes) to test exosomes derived from experimental tumor cell lines. The system also incorporates a new exosome labeling, isolation, and tracking approach using stable fluorescent carbocyanine dyes. The rationale for establishing our system stems in part from the technical challenges of dealing with the exosome constructs themselves, which are very difficult to isolate and apply in large quantities even in vitro, much less in vivo, in tissue angiogenesis studies. Given that exosomal constituents and activities are likely to change over time as the tumor cell itself adapts to local environments and the selective pressure of therapeutics, a rapid assay of in vitro activity of patient-derived exosomes could be invaluable for clinical therapeutic decision making. An in vitro assay also would allow conjunctive readouts such as cytokine or RNA microarray analyses of sprouting endothelial cells.

We show for the first time that melanoma exosomes can be successfully labeled, isolated, and tracked using fluorescent carbocyanine dyes. Tracking of melanoma exosomes within endothelial cell cultures reveals a novel association between exosomes and cellular nanotubes. Moreover, the endothelial response to melanoma exosomes is a complicated phenomenon that exhibits both trophic and proliferative changes in a dose-dependent manner, which simultaneously modulates both angiogenic and immunological cytokine signaling. The implication is that the melanoma 'exosomal messenger system' is capable of multifunctional paracrine bioactivities that facilitate tumor communication at a distance, especially in the matter of turf preparation, which could be assessed in vitro for individual tumor types from single sources.

\section{MATERIALS AND METHODS Materials and Cell Culture}

The 2F-2B mouse endothelial cell line was purchased from ATCC (CRL-2168) and maintained in 2D culture using Dulbecco's modified Eagle's medium (DMEM) with $4.5 \mathrm{~g} / \mathrm{l}$ glucose, 90\%; heat-inactivated fetal bovine serum, $10 \%$ at $37^{\circ} \mathrm{C}$; and $5 \% \mathrm{CO}_{2} .2 \mathrm{~F}-2 \mathrm{~B}$ cells were cultured using EGM-2 media (Clonetics catalog CC-3162) for 3D spheroid assays. Mouse B16-F10 melanoma cells were purchased from ATCC and maintained in culture using 90\% DMEM; $10 \%$ heatinactivated fetal bovine serum at $37^{\circ} \mathrm{C}$; and $5 \% \mathrm{CO}_{2}$.

\section{Isolation of Exosomes}

B16-F10 melanoma or 2F-2B endothelial cell cultures were grown to $70 \%$ confluence in three $300 \mathrm{~cm}^{2}$ flasks. Culture media was removed and cells washed in PBS. Cells were cultured for $48 \mathrm{hrs}$ in the presence of conditioned media. Conditioned culture media was prepared by subjecting normal culture media to overnight ultracentrifugation at $100000 \mathrm{~g}$ to remove bovine exosomes. ${ }^{11}$ B16 melanoma exosomes were collected from $48 \mathrm{~h}$ culture in conditioned media through standard differential centrifugation steps using a $70 \mathrm{Ti}$ rotor. ${ }^{11}$ Culture media was spun and supernatants collected from $300 \mathrm{~g}$ for $10 \mathrm{~min}, 2000 \mathrm{~g}$ for $10 \mathrm{~min}$, to remove residual cells and debris, $10000 \mathrm{~g}$ for $30 \mathrm{~min}$ to remove microparticles, ${ }^{12}$ and $100000 \mathrm{~g}$ for $2 \mathrm{~h}$ (in the presence or absence of $1.0 \mu \mathrm{M}$ DiI $\left(1,1^{\prime}\right.$-dioctadecyl-3,3,3' $3^{\prime}$ tetramethylindocarbocyanine perchlorate) or DiR $\left(1,1^{\prime}\right.$ dioctadecyl-3,3,3' $3^{\prime}$-tetramethylindotricarbocyanine iodide) (Invitrogen)) to pellet exosomes. Exosome pellets were washed thrice in PBS, pooled, and re-isolated in PBS at $100000 \mathrm{~g}$ for $2 \mathrm{~h}$. Exosome pellets were resuspended in $1 \mathrm{ml}$ of PBS, protein content measured by BCA absorbance (Pierce) and stored at $-80^{\circ} \mathrm{C}$ until use.

\section{Flotation of Exosomes on a Continuous Sucrose Gradient}

Flotation of exosomes on a continuous sucrose gradient (2.0-0.25 M sucrose, $20 \mathrm{mM}$ HEPES/ $\mathrm{NaOH}, \mathrm{pH} 7.4)$ was performed similarly as previously described, but using an SW 41 rotor. ${ }^{13}$ The gradient (prepared using a Hoefer gradient maker) was spun for $>15 \mathrm{~h}$ at $100000 \mathrm{~g}$ and $111 \mathrm{ml}$ fractions were collected from the bottom up. The density of each fraction was calculated using a refractometer. ${ }^{11} \mathrm{~A}$ total of $50 \mu \mathrm{l}$ of each fraction was added to a black 96-well plate and DiR exosome fluorescence detected using a Xenogen IVIS fluorescent imager (Caliper Life Sciences). Each of the remaining fractions was resuspended in $24 \mathrm{ml}$ of PBS and recentrifuged for $2 \mathrm{~h}$ at $200000 \mathrm{~g}$ to pellet exosomes. The protein level of each fraction was measured by BCA absorbance (Pierce), and exosome pellet was diluted in Laemmli's sample buffer and run on a $4-20 \%$ Tris-glycine gel (Bio-Rad). Exosomal proteins were detected by western blot using anti-Melan A antibody (Santa Cruz Biotechnology) and antiCalnexin (Millipore).

\section{Electron Microscopy}

Purified exosome pellets were fixed using $2.5 \%$ glutaraldehyde in PBS for $30 \mathrm{~min}$ on ice. After rinsing, the pellet was sequentially stained with osmium tetroxide and uranyl acetate, then dehydrated and embedded in Polybed 812. Tissue was thin sectioned on a Reichert-Jung Ultracut, viewed on a Zeiss 902 Electron Microscope, and recorded with Kodak EM film. EM reagents were purchased from Electron Microscopy Sciences.

\section{Fumagillin Nanoparticle Formulation}

Perfluorocarbon nanoparticles were prepared as described previously. ${ }^{14,15}$ Briefly, the emulsions comprised $20 \%$ (vol/ vol) perfluoro-15-crown-5 (Exfluor Research Corporation), $2 \%(\mathrm{wt} / \mathrm{vol})$ of a surfactant comixture, $1.7 \%(\mathrm{wt} / \mathrm{vol})$ glycerin, and water for the balance. The surfactant comixture included 98 mole percent lecithin (Avanti Polar Lipids, Inc.), 1.8 mole percent phosphatidylethanolamine (Avanti Polar Lipids, Inc.), and chloroform:methanol mixture in the ratio 
3:1, which was dried to a lipid film under vacuum. Nanoparticle formulations included 0.2 mole percent fumagillin (a gift from the National Cancer Institute), which was added to the surfactant mixture at the proportionate expense of lecithin. The surfactant components were prepared as published, ${ }^{14,15}$ combined with $\mathrm{PFOB}$ and distilled deionized water, and emulsified (Microfluidics) at 20000 psi for $4 \mathrm{~min}$. Particle sizes were determined at $37^{\circ} \mathrm{C}$ using a laser light scattering submicron particle analyzer (Brookhaven Instruments).

\section{Spheroid Angiogenesis Assay}

2F-2B cells were cultured two-dimensionally in $75 \mathrm{~cm}^{2}$ culture flask, isolated, and applied to the wells of a 48-well (Corning) culture plate (50000 cells per well) containing $200 \mu \mathrm{l}$ of prepared $1 \times$ matrigel (BD Biosciences) per well and $1 \mathrm{ml}$ of EGM-2 culture media. When used, 2F-2B or B16 melanoma exosomes were applied to the endothelial culture wells at 0 , $2.5,5$, or $10 \mu \mathrm{g} / \mathrm{ml}$ protein concentrations. Spheroids were allowed to develop at $37^{\circ} \mathrm{C}$ and $5 \% \mathrm{CO}_{2}$, and were observed through phase contrast microscopy using a Nikon Diaphot 300 microscope and images were recorded using a Basler A302fc microscope camera in conjunction with Vision Assist software for the number and size of spheroids as well as the presence or absence of endothelial sprouts. Sizes and numbers of spheroids were computed in randomly selected highpowered fields of view by ImageJ analysis software. ${ }^{16}$

\section{Fluorescent and Confocal Microscopy}

2F-2B endothelial cells (100000 cells per chamber) were cultured on matrigel $(200 \mu \mathrm{l})$, in the presence or absence of $20 \mu \mathrm{g} / \mathrm{ml}$ of fluorescent red DiI-labeled $(1 \mu \mathrm{M}) 2 \mathrm{~F}-2 \mathrm{~B}$ or B16 melanoma exosomes for $24 \mathrm{~h}$, coating the bottom of a 4 chamber microscopy culture slide $\left(\mathrm{NUNC}^{(\mathrm{TM})}\right.$ Lab-Tek $^{(\mathrm{TM})}$ Chamber Slide ${ }^{(\mathrm{TM})}$ System). After $24 \mathrm{~h}$ of incubation, endothelial tubules were fixed for $10 \mathrm{~min}$ with $3.7 \%$ paraformaldehyde, washed thrice with PBS, and extracted with acetone at $-20^{\circ} \mathrm{C}$ for $5 \mathrm{~min}$ and blocked with $1 \%$ BSA in PBS for $30 \mathrm{~min}$. Tubules were then washed thrice with PBS and stained with AlexaFluor 488 phalloidin, according to manufacturer's protocol (Invitrogen). Briefly, two units of methanolic stock solution was diluted into $200 \mu \mathrm{l}$ of PBS containing $1 \%$ BSA and added to each slide chamber containing tubules for $20 \mathrm{~min}$ at room temperature to stain actin cytoskeleton green. Chamber slides were then washed thrice with PBS and sealed with DAPI (stains nuclei fluorescent blue) VECTASHIELD mounting media (Vector Laboratories, Inc.). Slides were then visualized using an Olympus BX61 fluorescent or confocal (Zeiss Meta 510, Thornwood, NY, USA) microscope using standard filter sets.

\section{Cytokine Array Analysis}

$2 \mathrm{~F}-2 \mathrm{~B}$ endothelial cells were cultured for $72 \mathrm{~h}$ to form spheroids followed by the addition of $0,2.5$, or $10 \mu \mathrm{g} / \mathrm{ml} \mathrm{B16}$ melanoma exosomes for 10 days to produce cytokines in the spheroid culture media. Three sets of culture media samples were collected and pooled for each exosome dosing group and analyzed using two different cytokine arrays: (1) TransSignal Mouse Angiogenesis array (Panomics, Fremont, CA, USA) and (2) Mouse Proteome Profiler array (R\&D Systems, Minneapolis, MN, USA), following the manufacturer's instructions provided with the kits. Fluorescent signals were processed using Kodak BioMax light radiographic film for Streptavidin-HRP-based chemiluminescent detection. Film results were scanned and digitized using a Hewlett Packard Scanjet 3970 flatbed scanner. Individual cytokine signal intensities were evaluated using ImageJ analysis software. ${ }^{16}$ Results are reported as average cytokine signal intensity based on pixel density.

\section{Statistics}

For spheroid sizing and cell proliferation, ANOVA was used to calculate statistical significance $P<0.05$ between exosome treatment and control groups. For cytokine array analysis, the absolute value of the average percentage change (average of 2.5 and $10 \mu \mathrm{g} / \mathrm{ml})$ over normalized control $(0 \mu \mathrm{g} / \mathrm{ml}$ of exosomes) was calculated for each cytokine. Cytokine levels that changed more than $10 \%$ from the average changes of absolute value as compared with a normalized control group were considered to be affected by the experimental procedures, according to ANOVA that revealed statistical significance $(P<0.05)$ for difference from control in each case.

\section{RESULTS \\ Development of an Endothelial Spheroid Assay for Exosomal Activation}

The methods and assay system described herein recognize earlier work by Haspel et al. ${ }^{17}$ and Stahl et al. ${ }^{18}$ but the present approaches exploit a newly discovered natural tendency of readily available standard cell types (2F-2B endothelial cells from ATCC) to spontaneously aggregate on matrigel to form 3D endothelial spheroids. These cells are derived from an SV40-transformed cell line originally generated for antiangiogenic pharmacology studies. ${ }^{19}$ The cell line expresses common endothelial markers as well as endothelial markers present on B16 melanoma and human tumor vasculature, making it ideally suited for angiogenic studies.

2F-2B mouse endothelial cell spheroids were created by culturing cells on matrigel. Surprisingly, 2F-2B cells exhibited a rare ability to spontaneously aggregate into uniformly distributed spheroids (Figure 1a) when placed on matrigel in contrast to other cell types, such as SMHEC4 endothelial cells, which require prolonged artificial pre-formation in round bottomed 96-well plates to induce spheroid formation. ${ }^{17}$ The individual $2 \mathrm{~F}-2 \mathrm{~B}$ endothelial cells migrate toward each other forming a lace-like network by $4 \mathrm{~h}$. By $24 \mathrm{~h}$, the cells have aggregated into thick tubules in the process of contracting into spheroids. By $72 \mathrm{~h}$, tubules have fully contracted leading to the formation of round spheroids. 

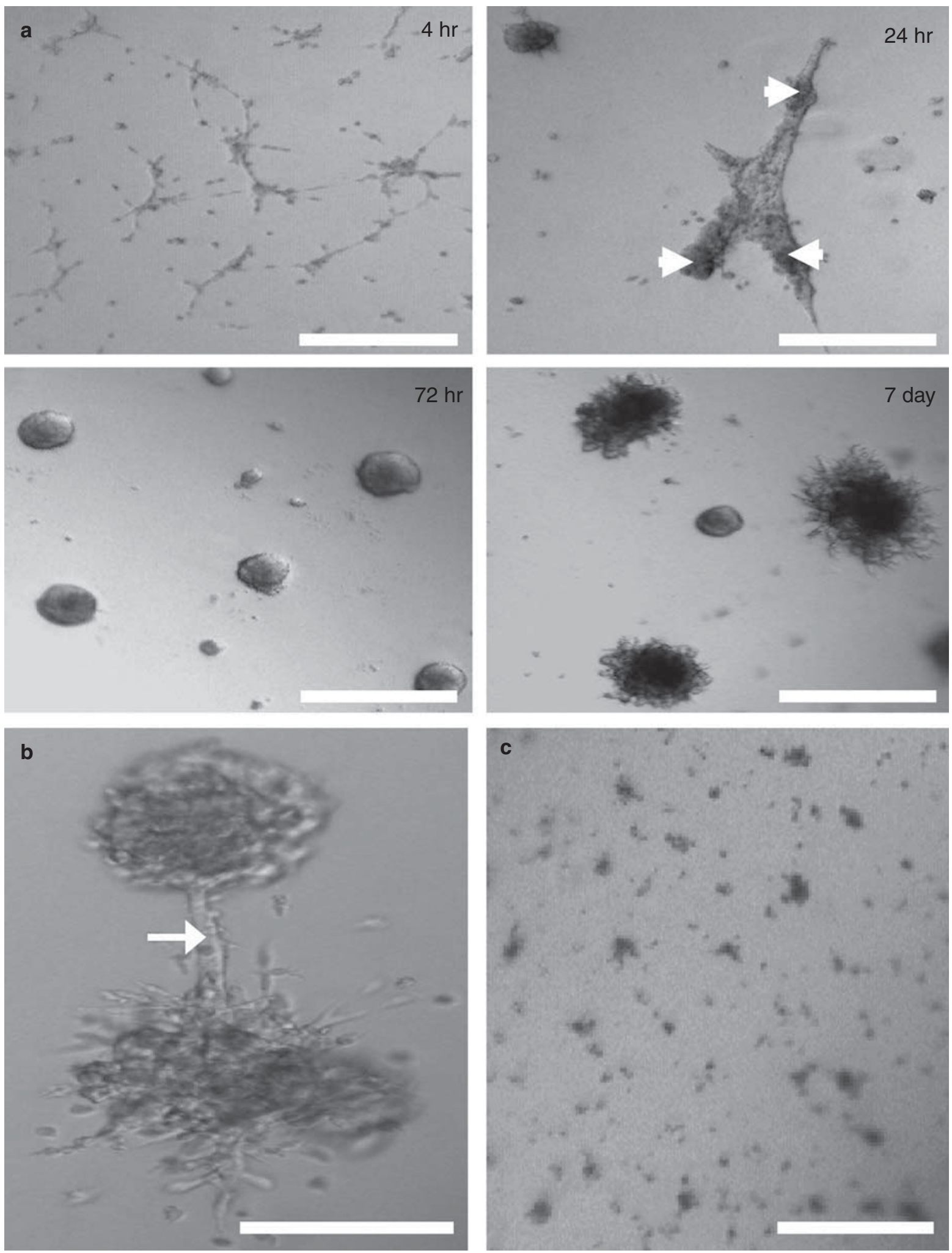

Figure 1 Endothelial cell lines, 2F-2B (50000 cells per well), spontaneously form spheroids on 3D matrigel as evidenced by phase contrast microscopy. (a) Cells migrate into a lace-like network by $4 \mathrm{~h}$. Endothelial tubules form and begin contracting into spheroids (arrowheads) by $24 \mathrm{~h}$. Formation of spheroids occurs after $72 \mathrm{~h}$ in culture. Circumferential spheroid sprouting is observed by 7 days. (b) Beyond 7-day culture, spheroid sprouts can form anastomotic connections (arrow). (c) In the presence of antiangiogenic fumagillin nanoparticles for $72 \mathrm{~h}$ (final culture fumagillin concentration $=4 \mu \mathrm{M}$ ), spheroids fail to develop. Bar $=200 \mu \mathrm{m}$. 
Looking ahead to 7 days, spheroids develop numerous 'capillary-like' sprouts around their circumference. Some of the sprouts from adjacent spheroids will form anastomotic junctions of communication (Figure 1b).

Having successfully generated endothelial spheroids, we next sought to assess whether they respond to a potent antiangiogenic compound by growing the spheroids in the presence of fumagillin. Fumagillin is a mycotoxin produced by Aspergillus fumigatus, ${ }^{14,15}$ and is a potent inhibitor of angiogenesis operating by targeting the methionine aminopeptidase- 2 of endothelial cells. ${ }^{20}$ We have previously shown that fumagillin itself, which is hydrophobic, can be incorporated into the lipid shell of a perfluorocarbon-core nanoparticle to overcome its poor solubility in aqueous solutions and allow for concentrated delivery to angiogenic blood vessels that are formed in various pathologies, resulting in their marked suppression. ${ }^{14,15}$ Thus, when $2 \mathrm{~F}-2 \mathrm{~B}$ cells were treated with antiangiogenic fumagillin nanoparticles for $72 \mathrm{~h}$, spheroids did not form in 3D cultures, which validate the proposed assay for assessing angiogenesis induction (Figure 1c).

\section{Methods for Isolation of Tumor Exosomes In Vitro}

On the basis of current methodology, exosomes can be differentiated from other membrane particulate matter according to three criteria: (1) size $(\sim 30-100 \mathrm{~nm}),(2)$ unique flotation density $(1.22-1.08 \mathrm{~g} / \mathrm{ml})$ on continuous sucrose gradients, ${ }^{11-13,21-23}$ and (3) characteristic 'cup-shaped' morphology through electron microscopy versus black 'electrondense' microparticles. $^{21}$ Mouse B16 melanoma cells were cultured and exosomes isolated by differential centrifugation (Figure 2) ${ }^{11}$ Although the standard approach for verification of exosome isolation relies on electron microscopy, ${ }^{11} \mathrm{dy}$ namic light scattering (Brookhaven Instruments) was used to size B16 melanoma exosomes $(74 \pm 13 \mathrm{~nm}$ ) (Figure 2a) just before exosome pelleting at $100000 \mathrm{~g}$ (Figure 2b). We find that sizing exosomes after pellet formation results in erroneous results given the tendency for exosomes to clump. The measurement can be performed conveniently in as little as $10 \mathrm{~min}$, and greatly expedites confirmation of exosome isolation over traditional electron microscopy techniques (Figure 2d). Exosomes were pelleted (Figure 2b) at $100000 \mathrm{~g}$ for $2 \mathrm{~h}$ in the presence of DiI or DiR $(1 \mu \mathrm{M})$ to fluorescently label exosomes (DiI used in Figure $2 \mathrm{~b}$ and DiR used in Figure 2c). DiI or DiR are lipophilic fluorescent red (DiI) or near infrared (DiR) carbocyanin dyes that remain stable for days in living cultures and exosomes and undergo negligible transfer between intact membranes (Invitrogen), which allows it to remain associated with exosomes exclusively. To confirm the density of our B16 melanoma exosome population isolated by differential centrifugation, DiR-labeled B16 exosomes were separated on a continuous sucrose gradient. Given that melanoma exosomes contain very few of the standard exosomal protein markers, ${ }^{11}$ and exosome identification with markers may miss up to $50 \%$ of exosomes depending on the antibody used, ${ }^{12}$ we reasoned that tracking exosomes on sucrose gradients with DiR would expedite the process of determining exosome density. As shown, B16 melanoma exosomes isolated on a continuous sucrose gradient exhibit an exosome density between 1.10 and $1.21 \mathrm{~g} / \mathrm{ml}$ (Figure 2c). This exosome density has been confirmed by many laboratories to range from $\sim 1.08$ to $1.22 \mathrm{~g} / \mathrm{ml}$ for a variety or exosome types, ${ }^{11-13}$ including melanoma exosomes. ${ }^{22,23}$ Moreover, the position of the exosomes on the gradient directly corresponds to the protein content of the fractions. Finally, the B16 melanoma exosomes expressed one of the few unique melanoma exosomal markers (Melan A/ MART-1), ${ }^{11,24}$ and did not express the contaminant endoplasmic reticulum marker, calnexin. ${ }^{24}$

Finally, we confirmed isolation of B16 melanoma exosomes morphologically (Figure $2 \mathrm{~d}$ ) using electron microscopy. As shown, B16 exosomes isolated by differential centrifugation express the characteristic 'cup-shaped' morphology associated with exosomes ${ }^{21}$ and are $\sim 50-100 \mathrm{~nm}$ in diameter, validating our analysis by dynamic light scattering. Thus, with the use of these techniques, we demonstrate successful isolation of B16 melanoma exosomes at the correct size, density, and morphology reported for exosomes.

\section{Tumor Exosomes Engage Endothelial Tubule Networks in 3D}

Having successfully isolated melanoma exosomes, the next aim was to determine whether they interact with and influence the morphology of endothelial vasculature in our 3D culture model. 2F-2B (control) endothelial exosomes (Figure $3 \mathrm{~b}$ ) and B16 melanoma exosomes (Figures $3 \mathrm{c}$ and d) were fluorescently labeled and incubated with 3D-cultured $2 \mathrm{~F}-2 \mathrm{~B}$ endothelial cells for $24 \mathrm{~h}$ to compare their influence on endothelial tubule formation with non-exosome-treated tubules (Figure 3a). As shown in Figure 3, cell nuclei are stained with fluorescent blue DAPI (VECTASHIELD mounting media, Vector Labs, CA, USA), the connecting tubule networks are visualized with fluorescent green staining of the actin cytoskeleton using AlexaFluor 488 phalloidin (Invitrogen), and exosomes stained with DiI reveal tubule interactions.

We observed that exosomes interact with developing endothelial tubules in novel ways. Compared with controls (Figure 3a), melanoma exosomes (Figure 3c) alter the morphology of the tubule network in a manner that favors increased tubule branching, whereas $2 \mathrm{~F}-2 \mathrm{~B}$ exosomes (Figure 3b) appear to decrease tubule branching and connections. We conclude that exosomes readily interact with tubule membranes, because exosome signal appears within the cell bodies that make up connecting tubules and the developing spheroid cytoplasm (Figures $3 \mathrm{~b}$ and c). The location of the exosome signal does not coincide with the nucleus (Figures $3 \mathrm{~b}$ and c). However, colocalization of exosomes with cytoskeleton is observed for melanoma exosomes (Figures $3 \mathrm{c}$ and $\mathrm{d}$ ), but minimally with $2 \mathrm{~F}-2 \mathrm{~B}$ exosomes 
a b

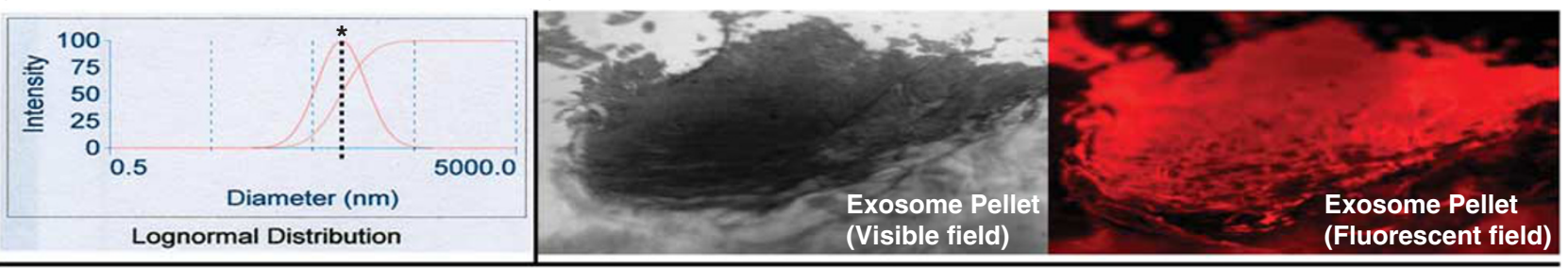

\begin{tabular}{|c|c|c|c|c|c|c|c|c|c|c|c|}
\hline Fraction \# & 1 & 2 & 3 & 4 & 5 & 6 & 7 & 8 & 9 & 10 & 11 \\
\hline Density $(\mathrm{g} / \mathrm{ml})$ & 1.26 & 1.26 & 1.26 & 1.21 & 1.14 & 1.10 & 1.08 & 1.07 & 1.06 & 1.04 & 1.03 \\
\hline $\begin{array}{l}\text { Exosome } \\
\text { Fluorescence } \\
\text { (DiR label) }\end{array}$ & & & & & & & & & & & \\
\hline Protein $(\mu \mathrm{g})$ & 0 & 0 & 0 & 16 & 40 & 3 & 0 & 0 & 0 & 0 & 0 \\
\hline
\end{tabular}

Calnexin

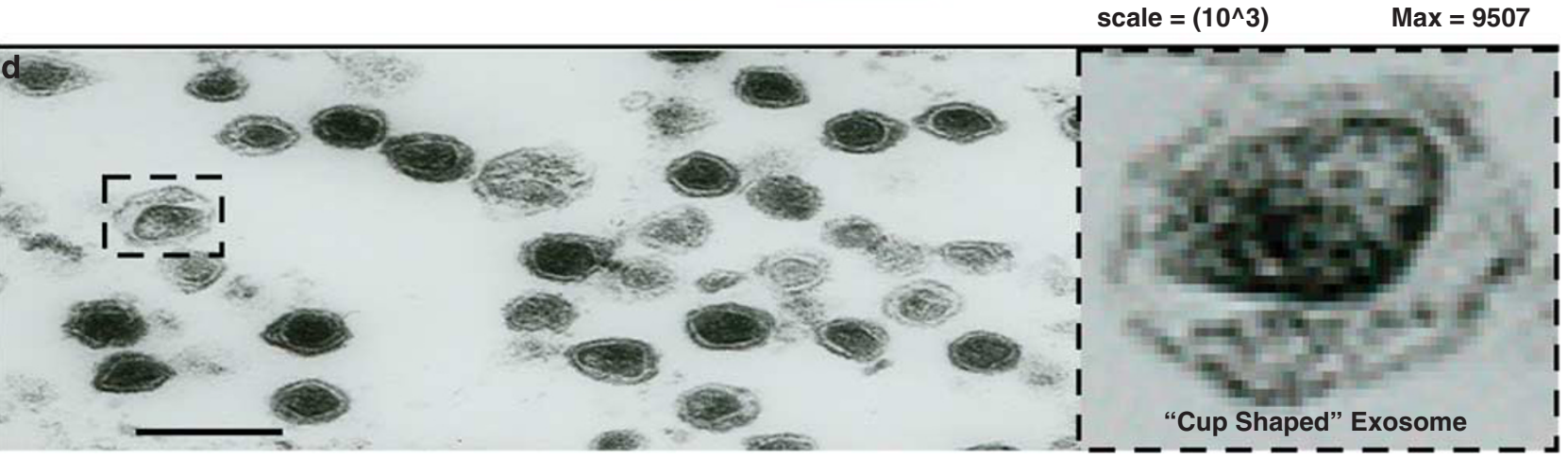

Figure 2 Isolation of B16-F10 melanoma exosomes. (a) Dynamic light scattering (DLS, Brookhaven Instruments) was used to confirm exosome isolation and the size of exosomes (before centrifugation at $100000 \mathrm{~g}$ to maximize accuracy by minimizing the measurement of exosome clusters). Exosome size distribution profile is represented as a bell-shaped curve. Dotted line crosses the logarithmic sizing curve at asterisk $(*)=74 \mathrm{~nm}(\mathrm{~s} . \mathrm{d} .=13 \mathrm{~nm}$, average of 24 individual measurements). (b) Exosome pellet visualized through a 70 Ti polycarbonate tube after differential centrifugation at $\times 20$ magnification. Exosomes were treated with Dil before pellet formation and display red fluorescence through fluorescent microscopy at $\times 20$ magnification. (c) DiR-labeled B16 melanoma exosome flotation on a continuous sucrose gradient (2.0-0.25 M). Arrow, single blue exosome band (black and white image, DiR is blue in visible light wavelengths) band present in gradient fraction 5 of the SW 41 centrifuge tube. Exosome fractions were further evaluated for protein content and expression of Melan A or calnexin marker proteins. (d) Transmission electron microscopy was used to confirm isolation of cup-shaped (see blow up) B16 melanoma exosomes. Various exosome sizes $\sim 50-100 \mathrm{~nm}$ are observed in concordance with DLS. Scale bar $=200 \mathrm{~nm}$.

(Figure 3b). This suggests differential compartmentalization of melanoma compared with $2 \mathrm{~F}-2 \mathrm{~B}$ exosomes in the cell.

The fluorescent signal for tumor exosomes acting as paracrine effectors appears different than the autocrine signal of endothelial exosomes. Melanoma exosome signal (Figure 3c) is both continuous throughout the tubule network and found in clusters, whereas endothelial exosome signal is found predominantly in clusters (Figure 3b). The clustering of exosomes at focal points within the tubule network (Figure $3 \mathrm{~b}$ ) suggests organization of the exosomes within organelle structures such as endosomes. The observation that melanoma exosome signal colocalizes with $\mathrm{f}$-actin and is present throughout anastomotic connections between adjoining cellular clusters supports the existence of a transport process for relaying exosomal messages between cells of the endothelial tubule network (Figure 3c). These data are consistent with the hypothesis that endothelial cells transport tumor exosomes between cells by way of tunneling nanotubule networks (TNTs) containing actin cytoskeleton (Figure 3d). TNT's were recently shown to transport HIV-1 particles, membrane vesicles, and endosomal organelles between cells, ${ }^{25-27}$ This has important implications on how tumors communicate with and influence vasculature for propagation and survival.

\section{Tumor Exosomes Induce Dose-Dependent Endothelial Spheroid Responses}

Having shown the ability of melanoma exosomes to interact with and promote endothelial tube formation over the course of $24 \mathrm{~h}$, the next experiments sought to determine the 
b

C

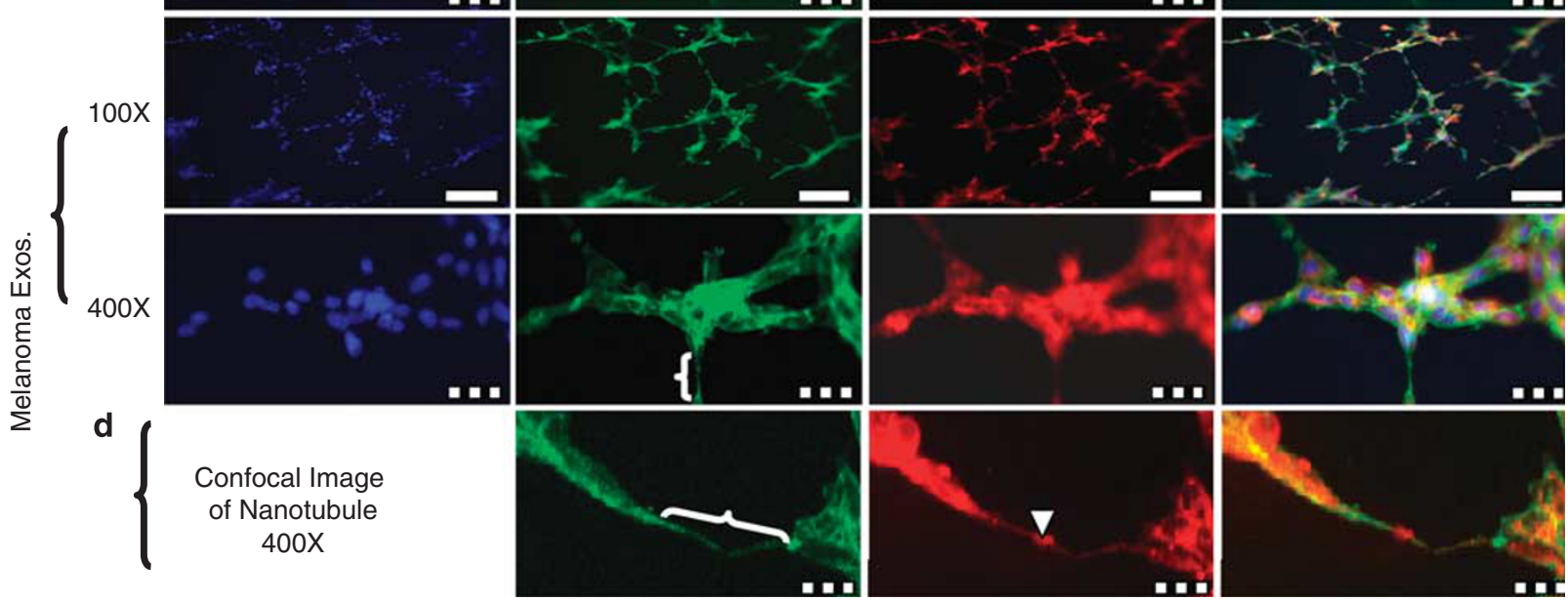

nuclei

100X
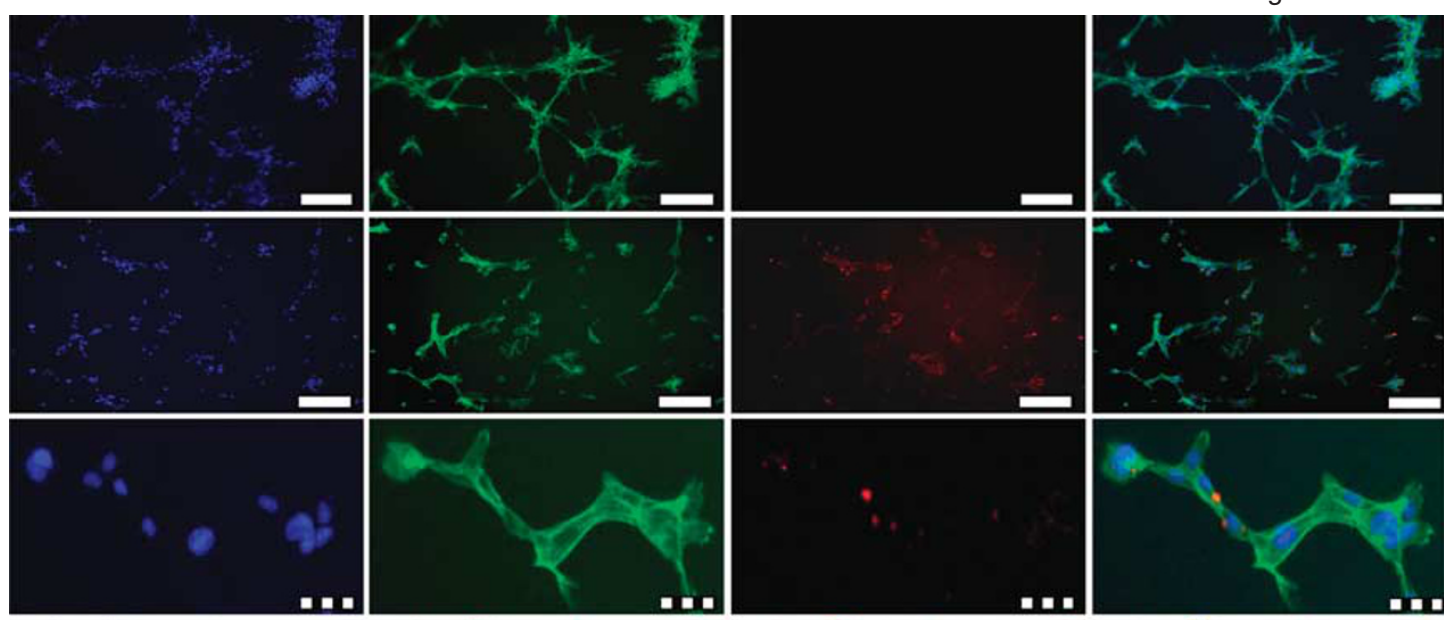

400X

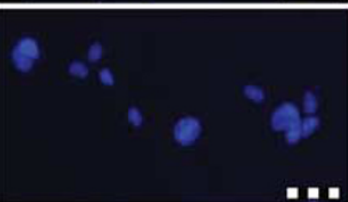

-
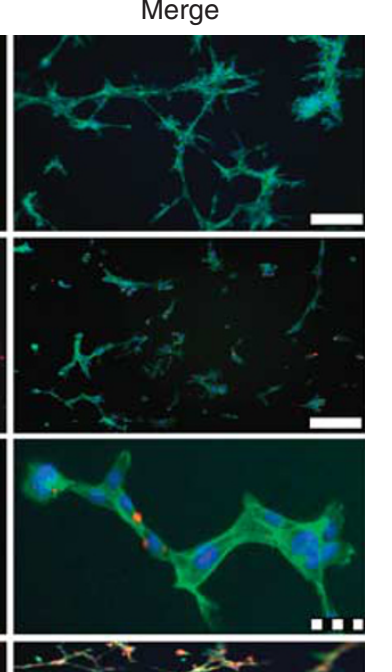

Figure 3 Exosomes interact with and influence the morphology of 3D 2F-2B endothelial cell tubules, as evidenced by fluorescent microscopy. (a) $2 \mathrm{~F}-2 \mathrm{~B}$ endothelial cells (100000 cells per chamber) cultured on matrigel for $24 \mathrm{~h}$ in the absence of exogenously labeled exosomes show no red background signal, whereas $\mathrm{f}$-actin is stained green. (b) Endothelial cells ( 100000 cells per chamber) cultured on matrigel for $24 \mathrm{~h}$ in the presence of $20 \mu \mathrm{g} / \mathrm{ml}$ of $2 \mathrm{~F}-2 \mathrm{~B}$ endothelial cell exosomes show decreased tubule branching versus that seen in pannel a and show exosome signal in red clusters within green $\mathrm{f}$-actinstained endothelial tubules. (c) Endothelial cells (100000 cells per chamber) cultured on matrigel for $24 \mathrm{~h}$ in the presence of $20 \mu \mathrm{g} / \mathrm{ml}$ of B16 melanoma exosomes show increased tubule branching versus that seen in pannel a and show colocalization between exosome signal in red clusters and green f-actinstained endothelial tubules. (d) Confocal image of red exosome signal colocalized with green-stained f-actin in a nanotube bridging two endothelial tubules. White flower brackets show nanotubes; arrowhead points toward exosome cluster within a nanotube; solid white bar, $200 \mu \mathrm{m}$; hatched bar, $40 \mu \mathrm{m}$. Blue fluorescence, DAPI stain (Vector labs); green, AlexaFluor 488 phalloidin stain (Invitrogen); red, Dil dye (Invitrogen).

long-term effects of melanoma exosomes on spheroid development. It is known that exosomes can modulate tissue patterning, ${ }^{3}$ which is a permanent effect, and, therefore, we hypothesized that once exposed to melanoma exosomes, the endothelial cells would undergo a long-term change, as evidenced by their pattern of cytokine expression. Thus, endothelial spheroids were developed for $72 \mathrm{~h}$ followed by application of a one-time dose of a range of exosome concentrations between 0 and $10 \mu \mathrm{g} / \mathrm{ml}$ for 10 days. It is important to note that we did not expect melanoma exosomes to be present in culture after 10 days after their initial application on day 1 . Instead, we were interested in the permanence of the changes induced by the melanoma exosomes over time. Spheroid morphology, number, and proliferation were then assessed (Figure 4). Distinct morphological differences are easily observed through microscopic visualization of representative spheroids by 13 days (Figure $4 \mathrm{a}$ ) and, thus, provide a visual way to coordinate timing for subsequent analyses, such as spheroid proliferation or cytokine production. Long-term culture of native $2 \mathrm{~F}-2 \mathrm{~B}$ spheroids (Figure 4a) produced fine branching 'needle-like' endothelial sprouts as seen on aortic ring models. ${ }^{28}$ However, in the presence of $2.5 \mu \mathrm{g} / \mathrm{ml}$ of melanoma exosomes, spheroid sprouts exhibited increased complexity in terms of size and number (Figure 4a). Increasing the exosome concentration to 5 or $10 \mu \mathrm{g} / \mathrm{ml}$ resulted in suppressed formation of the larger spheroid bodies (excluding circumferential tubules) in favor of more numerous smaller ones (Figures $4 \mathrm{a}$ and $\mathrm{b}$ ) that 
a

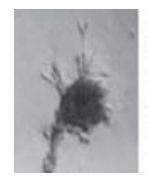

$0 \mu \mathrm{g} / \mathrm{ml}$

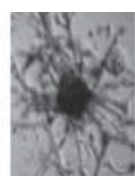

2.5

b

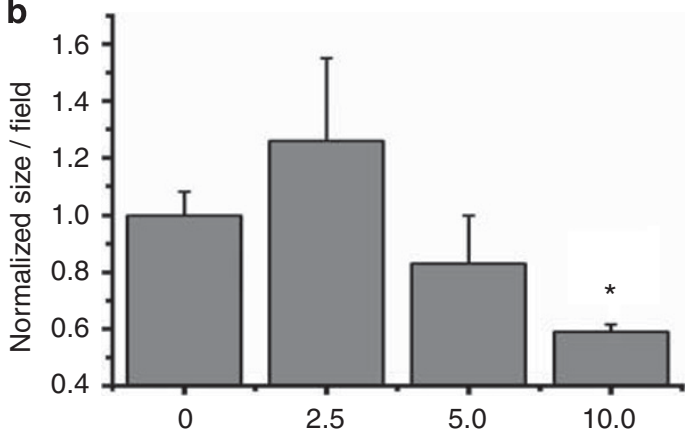

c

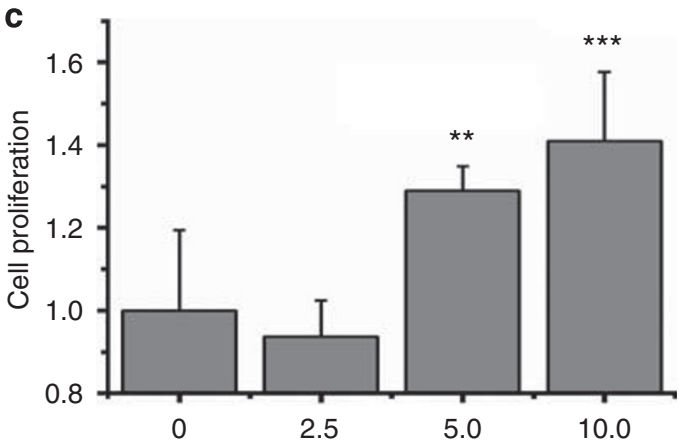

Figure 4 Long-term effects of varying B16-F10 melanoma exosome concentrations on $3 \mathrm{D}$ endothelial culture. $2 \mathrm{~F}-2 \mathrm{~B}$ endothelial cells ( 50000 cells per well) were cultured for $72 \mathrm{~h}$ to form spheroids followed by the addition of $0,2.5,5$, or $10 \mu \mathrm{g} / \mathrm{ml}$ of B16 exosomes for 10 days to study the permanence of exosome effects on spheroid development. (a) Phase contrast microscopy of representative spheroid morphology for each exosome dose. (b) ImageJ software was used to measure the area of individual spheroids in $\times 40$ magnification fields (see Supplemental information, Supplemental Figure S1). Average spheroid size per field was normalized against control $(0 \mu \mathrm{g})$. ANOVA was used to calculate significance for $P<0.05$ for a control of $N=48 ;{ }^{*} P=5.4 \times 10^{-5}$. Error bars represent the standard deviation of the average spheroid size from three random fields from different cultures. (c) XTT (tetrazolium salt) colorimetric reagent (MD Biosciences, St. Paul, MN, USA) was used to measure cell proliferation. Average endothelial cell proliferation was normalized against control $(0 \mu \mathrm{g}$ exosomes). ANOVA was used to calculate significance for $P<0.05$ for a control of $N=4 ;{ }^{* * P}=5.9 \times 10^{-3}$ and ${ }^{* * * P}=2.9 \times 10^{-3}$. Error bars represent the standard deviation of $N=4$ samples.

manifested less endothelial sprouting (Figure 4a) and quantitatively increased proliferation (Figure 4c). This observed bimodal dose-dependent response to exosome stimulation indicates that spheroids exhibit sprout enlargement at lower concentrations; yet at higher exosome concentrations, the rate of spheroid enlargement and sprouting is augmented with smaller more numerous spheroids.

These morphological data provide evidence to support the hypothesis that the differential response to tumor exosomes is attributable to trophic signaling at lower doses, but proliferative signaling (in less mature spheroids) at higher doses. Taken together, these data suggest that at lower concentrations, melanoma exosomes could promote spheroid maturation and sprouting angiogenesis leading to anastomotic communication networks. At higher concentrations, melanoma exosomes may favor increased endothelial proliferation inducing smaller, more numerous, and less mature-appearing spheroids.

\section{Cytokine Signaling Induced by Exosomes}

In view of the previously reported effect of tumor growth on downregulation of endothelial inflammatory biomarkers (for example, vascular adhesion molecules) that may facilitate escape from immune surveillance, ${ }^{6,7}$ and the established role of cytokines in tumor endothelial cell communication, ${ }^{29}$ we sought to delineate the effect of melanoma exosomes on endothelial inflammatory cytokine production. ${ }^{10}$ Although melanoma exosomes induce a dose-dependent bimodal influence on spheroid formation as shown by our assay (Figure 4), we further hypothesized that exosomes might simultaneously suppress endothelial production of pro-inflammatory cytokines. Accordingly, endothelial spheroids were developed for $72 \mathrm{~h}$ on matrigel followed by the addition of $0,2.5$, or $10 \mu \mathrm{g} / \mathrm{ml}$ of melanoma exosomes for 10 days. Spheroid culture supernatants were then collected and analyzed using angiogenic (Panomics mouse angiogenesis array) (Figure 5a) and inflammatory cytokine (mouse proteome profiler array (R\&D Systems) (Figures $5 \mathrm{~b}$ and $\mathrm{c}$ ) arrays.

In general, we observed that the cytokine expression patterns could be subdivided into 'concordant' (Figure 5b) and 'discordant' responses (Figures $5 \mathrm{a}$ and $\mathrm{c}$ ). Concordant cytokines are either unidirectionally increased (Figure 5b), regardless of the exosome dosing, or undergo bidirectional regulation within the discordant groups (Figures $5 \mathrm{a}$ and c). Overall, cytokines that might be considered proangiogenic increased with increasing exosome dosing: for example, IL$1 \alpha$, FGF, GCS-F, TNF $\alpha$, Leptin, TGF $\alpha$, and VEGF (Figure 5a). Interestingly, some inflammatory cytokines were suppressed at the higher exosome dose (Figure 5c), which could mimic the situation in vivo when angiogenesis is stimulated in the face of reduced expression of vascular adhesion molecules. ${ }^{30}$ For example, reduced expression of the pro-inflammatory cytokines TREM- $1,{ }^{31}$ I-TAC, ${ }^{32}$ IL-3, ${ }^{33}$ and IL- $16^{32}$ was observed at the $10 \mu \mathrm{g} / \mathrm{ml}$ dose of exosomes. Overall, these data provide supporting evidence for exosomal stimulation of endothelial angiogenic responses.

\section{DISCUSSION}

Angiogenesis is modulated not only by soluble angiogenic factors but also by cell-derived microparticles, microvesicles, and exosomes. Important distinctions exist in the composition, analysis, and bioactivity, and use of these types of particles that require consideration when interpreting the 

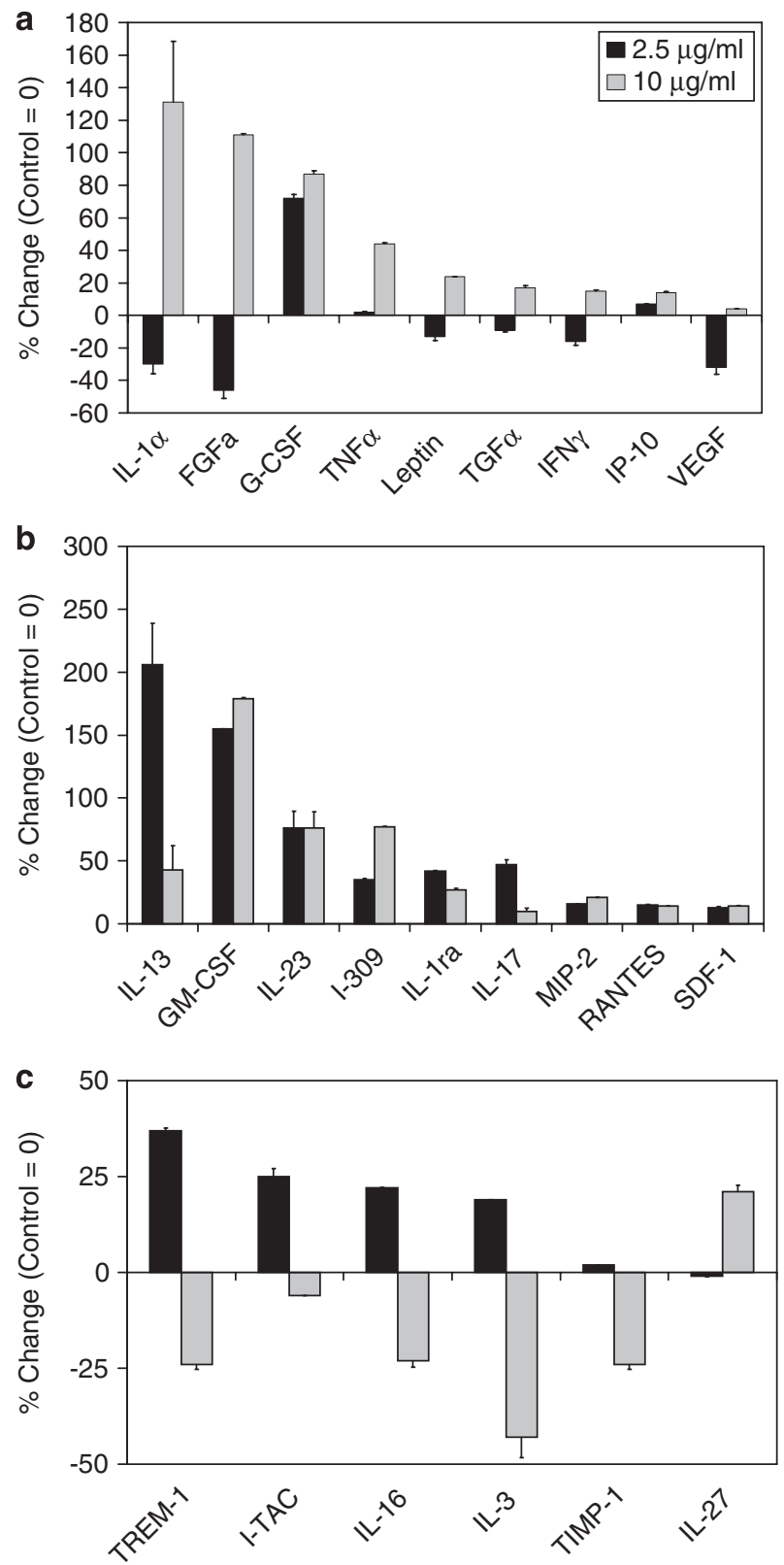

Figure 5 Melanoma exosomes modulate endothelial cytokine production. $2 \mathrm{~F}-2 \mathrm{~B}$ endothelial cells ( 50000 cells per well) were cultured for $72 \mathrm{~h}$ to form spheroids followed by the addition of $0,2.5$, or $10 \mu \mathrm{g} / \mathrm{ml}$ for 10 days to produce cytokines in the spheroid culture media. Three sets of culture media samples were collected and pooled for each exosome dosing group, and analyzed using cytokine arrays. (a) TransSignal Mouse Angiogenesis Antibody Array (Panomics) or (b and c) Proteome Profiler Mouse Cytokine Array (R\&D Systems). Cytokines levels are represented as a percent change over control (control $=0$ ). The absolute change in level for each cytokine group ( 2.5 and $10 \mu \mathrm{g} / \mathrm{ml}$ melanoma exosomes) was averaged. The average of absolute changes for all cytokines was $41 \%$. ANOVA analysis was used to calculate statistical significance. Cytokine levels that changed more than $10 \%$ from the absolute value average changes as compared with a normalized control group were considered to be affected by the experimental procedures, according to ANOVA that revealed statistical significance $(P<0.05)$ for difference from control in each case. The $P$-values for 2.5, 10, and combined concentrations are $0.035,0.016$, and 0.001, respectively. Error bars $=$ s.e.m. of replicates present on the arrays. present data. Microparticles comprise a heterogeneous population of particles $100-1000 \mathrm{~nm}$ in dimension. They are formed by a regulated reverse budding mechanism in which the plasma membrane blebs outward through reorganization of the underlying cortical actin cytoskeleton. ${ }^{34}$ This results in the direct detachment of plasma membrane buds into the extracellular space. Thus, microparticles represent one form of 'shedding vesicle. ${ }^{35}$ Microparticles can be separated from exosomes during differential centrifugation of cell culture supernatant between 10000 and $14000 g^{12,33,36}$

Platelet microparticles (PMPs), and to a lesser extent endothelial microparticles (EMPs), are arguably the most studied shedding vesicles, in large part, a result of their pronounced role in mediating coagulation and thrombosis. ${ }^{37-40} \mathrm{~A}$ number of studies have shown a role for PMPs in promoting endothelial angiogenesis both in vitro and in vivo. ${ }^{28,41,42}$ In contrast, the influence of EMPs on angiogenesis has been mixed with some studies reporting increased endothelial tubulogenesis, ${ }^{36}$ or impaired endothelial proliferation and increased apoptosis ${ }^{43}$ in vitro. Lymphocyte microparticles have also been shown to suppress angiogenesis in vitro and in vivo by interfering with VEGF signaling. ${ }^{44}$

Microvesicles (microparticles + exosomes) derived from platelets induce VEGF mRNA expression in lung cancer cells and promote their adherence to endothelial cells. ${ }^{45}$ Microvesicles derived from cancer cells, such as fibrosarcoma, prostate carcinoma, ${ }^{46}$ and ovarian carcinoma ${ }^{47}$ also promote angiogenesis. Ovarian cancer microvesicles have been reported to carry VEGF. ${ }^{48}$ Glioblastoma microvesicles promote endothelial proliferation in vitro. ${ }^{49}$ In contrast to what is known about microparticle- and microvesicle (microparticle + exosome)-mediated angiogenic effects, much less is known about tumor exosome-mediated angiogenesis alone. One report shows intercellular transfer of a truncated form of epidermal growth factor receptor (EGFRvIII) from an EGFRvIII-expressing cell line to a non-EGFRvIII-expressing cell line through exosomes. ${ }^{50}$ As VEGF gene expression is regulated by EGFRvIII, such receptor transfer may contribute to angiogenic signaling on tumor cell subsets. In addition, exosomes derived from a D6.1A tetraspanin-transfected pancreatic cancer cell line promote D6.1A-mediated endothelial tubulogenesis. ${ }^{51}$

In our investigations, we have created a powerful and efficient technique using dynamic light scattering in conjunction with fluorescent labeling to rapidly and efficiently isolate and track exosomes. This methodology can be easily adapted to a variety of in vitro and in vivo exosome studies. Dynamic light scattering provides for rapid and accurate exosome sizing during purification steps. In contrast to standard confirmation of exosome isolation by electron microscopy, it is less time consuming (minutes versus days), not as technically challenging, and does not result in any sample loss. This is very important for small volumes of human fluid samples wherein one cannot afford to spare exosomes to confirm their isolation. 
Labeling exosomes with fluorescent carbocyanine dyes before separation on sucrose gradients allows for distinct exosome subpopulations present in a variety of culture or body fluids to be efficiently separated from one another without exploring for markers, which may fluctuate on given populations depending on signaling or evolving pathological conditions or be lacking in specific exosome subsets. Such is the case with the platelet exosome marker, CD63. CD63 can be used to distinguish platelet-derived exosomes from PMPs. ${ }^{12}$ However, CD63 is only expressed on $\sim 50 \%$ of platelet exosomes making it possible to overlook non-CD63bearing exosomes during exosome isolation. Our technique would not miss non-CD63-bearing exosomes and may discover unknown subsets.

Historically, the first use of sucrose gradient preparations of exosomes by Raposo et al. ${ }^{13}$ was to determine whether MHC II proteins were bound to exosomes. It is important to note that they determined their exosome pellet, isolated by differential centrifugation, to be free of plasma membrane contaminants before flotation on a sucrose gradient. ${ }^{13} \mathrm{Re}-$ gardless, their method established exosome density as a criteria for confirming exosome isolation. Isolation of exosomes by flotation density on continuous sucrose gradients has since been confirmed by many groups and is very consistent ranging from $\sim 1.08$ to $1.22 \mathrm{~g} / \mathrm{ml}$ for a variety of exosome types $^{11-13}$ including melanoma exosomes. ${ }^{22,23}$

We expanded on the original approach of Raposo et al. by tracking fluorescent-labeled exosomes on continuous sucrose gradients and confirmed that B16 melanoma exosomes isolated by differential centrifugation are in fact a relatively pure population of exosomes, as confirmed by their presence at a density between 1.21 and $1.10 \mathrm{~g} / \mathrm{ml}$ (fractions 4-6) (Figure 2c). Moreover, the B16 melanoma exosomes were found to express one of the few unique melanoma exosomal markers (Melan A/MART-1), ${ }^{11,24}$ and do not express the endoplasmic reticulum contaminant marker, calnexin. ${ }^{24}$ This technique is useful for processing small volumes of in vivo-derived human fluid samples for functional exosomes studies or potential therapeutic applications in which one cannot afford to spare exosomes to confirm their isolation through biomarkers. This further supports the high level of purity of B16 melanoma exosomes isolated by differential centrifugation and strongly validates our novel technique for fluorescently labeling, tracking, and confirming exosome isolation through gradient centrifugation.

In addition, we report for the first time the association of exosomes with cellular nanotube structures. This may have important implications for how normal or tumor-derived exosomes interact with and transfer their messages between cells. We also report a dramatic action of native (unmodified) melanoma exosomes on endothelial regulation, as evidenced by spheroid growth and the formation of early vasculature precursors such as endothelial sprouts. A dose dependency was observed that implies a bimodal dose-dependent trophic versus proliferative influence of melanoma exosomes that could have implications for further understanding fundamental mechanisms of the metastasis that accompany the tumor 'angiogenic switch', as described originally by Hanahan and Folkman. ${ }^{52}$ These data suggest that melanoma cells may be capable of effecting angiogenesis from a distance through a membrane messenger (exosomal) system in the absence of direct cell to cell contact with endothelial cells. Our data indicate that no exogenous driver of angiogenesis is required under these experimental conditions, and that native melanoma exosomes already are capable of these actions.

Our data further suggest that tumor cells might communicate with, and possibly control, normal cells and tissues at sites distal from the tumor using exosomal paracrine effects to manipulate cytokine expression profiles. The possibility of an indirect mechanism of exosome-mediated immunosuppression would be potentiated by the known direct role of tumor exosomes in suppression of tumor cells. ${ }^{6,53}$ Earlier studies have shown exosome-mediated immunosuppression of NK cells, ${ }^{6}$ apoptosis of anti-tumor $\mathrm{T}$ cells, induction of myeloid suppressor cells, and impairment of monocyte differentiation into tumor antigen-presenting dendritic cells. ${ }^{7}$ In view of the likelihood that the concentration of tumor exosomes should be greatest nearest the tumor, the immune damping effect might be expected to increase as anti-tumor cells move closer to the actual tumor site.

Although this in vitro angiogenesis assay system cannot fully represent the complex in vivo situation, it is known that the morphological and physiological behavior of cells cultured in 2D differs considerably from that of fully spatial 3D pathology in vivo. Consequently, over the last few years, a welcome shift toward development of fast and reliable 3D culture assay systems has emerged. ${ }^{54-56}$ These systems, although not a replacement for ultimately confirming findings in vivo, encompass the ease and reproducibility of $2 \mathrm{D}$ reductionist systems while better mimicking natural in vivo 3D tissue morphology. Only a few 3D angiogenesis model systems are available at present, but they require the purchase of expensive commercial kits. ${ }^{17,57}$ Many commercial kits contain no source cell standardization that would ensure reproducibility.

In this assay, spontaneous aggregation of $2 \mathrm{~F}-2 \mathrm{~B}$ cells obviates the time consuming and tedious step of forcing endothelial spheroid formation through week long culture in round bottomed 96-well plates ${ }^{17}$ or 2-day culture on microcarriers. ${ }^{58}$ The assay offers multilevel angiogenic analyses for assessing differentiation of endothelial cells into higher order angiogenic structures. For example, the assay (Figure 1) allows quantification of (1) 3D migration of endothelial cells, (2) formation of endothelial tubules, (3) contraction of endothelial tubules into early spheroids, (4) formation of sprouting spheroids, and (5) anastomosis of sprouts into capillary-like networks. Moreover, the evolution of these structures occurs unforced with a natural spontaneity within manageable points of time so that they can be studied independently of one another with the use of simple microscopic interrogation. 
Although we only tested one type of tumor exosome from melanoma cells, the assay paradigm should serve as a test bed for other types as well. It may be the case that not all exosomes would elicit endothelial proliferation, but this might be important to determine in vitro before launching a campaign of antiangiogenic therapy for example. It is also not established from these data exactly what is the stimulatory component of the exosome: the exosome itself or the effector molecules carried within. Clearly, an in-depth analysis of B16 melanoma contents (protein, RNA etc.) will be necessary to explain the mechanism of our observations. The small amount of literature available for cancer patients suggest background, 'normal' exosome concentrations well below that of circulating tumor exosomes. ${ }^{59}$ Healthy age-matched control patients have negligible quantities of exosomes in the circulation, whereas exosome levels progressively increase with cancer stage, indicating the likely dependence of these responses on tumor-specific exosomes. Further research will be required to tease out the nature of angiogenic exosome effectors, although exosomes are known to be replete with myriad potential candidates such as microRNA. ${ }^{59}$ In addition, other secreted vesicles, glioblastoma microvesicles, have been shown to stimulate the proliferation of a glioma cell line and transfer Gaussia luciferase (Gluc) reporter activity to human brain microvascular endothelial cells. ${ }^{49}$

In summary, tumor exosomes are capable of activating endothelial cells in a dose-dependent manner, which may afford cancer a means of communicating with, manipulating, and 'tuning' its host environment. It seems probable that a number of known cancer signaling pathways could be integrated in a compact exosomal vehicle, as the exosome is itself derived from the cancer cell, but may escape immune surveillance and circulate in mass quantities. A future goal will be to design and construct novel approaches to intercept and manipulate this nanoscale cancer communication system for the purposes of understanding the mechanisms it may use to promote metastasis.

Supplementary Information accompanies the paper on the Laboratory Investigation website (http://www.laboratoryinvestigation.org)

\section{DISCLOSURE/CONFLICT OF INTEREST}

The authors declare no conflict of interest.

1. Schorey JS, Bhatnagar S. Exosome function: from tumor immunology to pathogen biology. Traffic 2008;9:871-881.

2. Admyre $C$, Johansson SM, Paulie $S$, et al. Direct exosome stimulation of peripheral human T cells detected by ELISPOT. Eur J Immunol 2006:36:1772-1781.

3. Lakkaraju A, Rodriguez-Boulan E. Itinerant exosomes: emerging roles in cell and tissue polarity. Trends Cell Biol 2008;18:199-209.

4. lero $M$, Valenti $R$, Huber $V$, et al. Tumour-released exosomes and their implications in cancer immunity. Cell Death Differ 2008;15:80-88.

5. Taylor DD, Gercel-Taylor C. Tumour-derived exosomes and their role in cancer-associated T-cell signalling defects. $\mathrm{Br} \mathrm{J}$ Cancer 2005;92:305-311.
6. Liu C, Yu S, Zinn K, et al. Murine mammary carcinoma exosomes promote tumor growth by suppression of NK cell function. J Immunol 2006:176:1375-1385.

7. lero $M$, Valenti $R$, Huber $V$, et al. Tumour-released exosomes and their implications in cancer immunity. Cell Death Differ 2008;15:80-88.

8. Bidard F-C, Pierga J-Y, Vincent-Salomon A, et al. A 'class action' against the microenvironment: do cancer cells cooperate in metastasis? Cancer Metastasis Rev 2008;27:5-10.

9. Rinderknecht $M$, Detmar $M$. Tumor lymphangiogenesis and melanoma metastasis. J Cell Physiol 2008;216:347-354.

10. Mahabeleshwar GH, Byzova TV. Angiogenesis in melanoma. Semin Oncol 2007;34:555-565.

11. Thery C, Clayton A, Amigorena S, et al. Isolation and characterization of exosomes from cell culture supernatants and biological fluids. Curr Protoc Cell Biol 2006;3:3.22.21-23.22.29.

12. Heijnen HF, Schiel AE, Fijnheer R, et al. Activated platelets release two types of membrane vesicles: microvesicles by surface shedding and exosomes derived from exocytosis of multivesicular bodies and alpha-granules. Blood 1999;94:3791-3799.

13. Raposo G, Nijman HW, Stoorvogel W, et al. B lymphocytes secrete antigen-presenting vesicles. J Exp Med 1996;183:1161-1172.

14. Winter PM, Neubauer AM, Caruthers SD, et al. Endothelial $\alpha v \beta 3$ integrin-targeted fumagillin nanoparticles inhibit angiogenesis in atherosclerosis. Arterioscler Thromb Vasc Biol 2006;26:2103-2109.

15. Winter PM, Schmieder AH, Caruthers SD, et al. Minute dosages of $\alpha v \beta 3$ targeted fumagillin nanoparticles impair $\mathrm{Vx}-2$ tumor angiogensis and development in rabbits. FASEB J 2008:22:1-10.

16. Rasband WS. ImageJ. US National Institutes of Health: Bethesda, MD, USA\#\#http://rsb.info.nih.gov/ij/, , 1997-2008.

17. Haspel HC, Scicli GM, McMahon G, et al. Inhibition of vascular endothelial growth factor-associated tyrosine kinase activity with su5416 blocks sprouting in the microvascular endothelial cell spheroid model of angiogenesis. Microvasc Res 2002;63:304-315.

18. Stahl $A$, Wu $X$, Wenger $A$, et al. Endothelial progenitor cell sprouting in spheroid cultures is resistant to inhibition by osteoblasts: a model for bone replacement grafts. FEBS Lett 2005;579:5338-5342.

19. Walter-Yohrling J, Morgenbesser S, Rouleau C, et al. Murine endothelial cell lines as models of tumor endothelial cells. Clin Cancer Res 2004; 10:2179-2189.

20. Sin N, Meng L, Wang MQW, et al. The anti-angiogenic agent fumagillin covalently binds and inhibits the methionine aminopeptidase, MetAP2. Proc Natl Acad Sci 1997;94:6099-6103.

21. Thery $C$, Boussac $M$, Veron $P$, et al. Proteomic analysis of dendritic cellderived exosomes: a secreted subcellular compartment distinct from apoptotic vesicles. J Immunol 2001;166:7309-7318.

22. Wolfers J, Lozier A, Raposo G, et al. Tumor-derived exosomes are a source of shared tumor rejection antigens for CTL cross-priming Nat Med 2001;7:297-303.

23. Riteau B, Faure F, Menier C, et al. Exosomes bearing HLA-G are released by melanoma cells. Hum Immunol 2003;64:1064-1072.

24. Mears R, Craven RA, Hanrahan S, et al. Proteomic analysis of melanoma-derived exosomes by two-dimensional polyacrylamide gel electrophoresis and mass spectrometry. Proteomics 2004;4: 4019-4031.

25. Sowinski S, Jolly C, Berninghausen O, et al. Membrane nanotubes physically connect $T$ cells over long distances presenting a novel route for HIV-1 transmission. Nat Cell Biol 2008;10:211-219.

26. Gurke S, Barroso JF, Hodneland E, et al. Tunneling nanotube (TNT)-like structures facilitate a constitutive, actomyosin-dependent exchange of endocytic organelles between normal rat kidney cells. Exp Cell Res 2008;314:3669-3683.

27. Rustom A, Saffrich R, Markovic I, et al. Nanotubular highways for intercellular organelle transport. Science 2004;303:1007-1010.

28. Brill A, Dashevsky O, Rivo J, et al. Platelet-derived microparticles induce angiogenesis and stimulate post-ischemic revascularization. Cardiovasc Res 2005;67:30-38.

29. Lazar-Molnar $\mathrm{E}$, Hegyesi $\mathrm{H}$, Toth $\mathrm{S}$, et al. Autocrine and paracrine regulation by cytokines and growth factors in melanoma. Cytokine 2000;12:547-554.

30. Dirkx AEM, oude Egbrink MGA, Kuijpers MJE, et al. Tumor angiogenesis modulates leukocyte-vessel wall interactions in vivo by reducing endothelial adhesion molecule expression. Caner Res 2003;63: 2322-2329. 
31. Chen LC, Laskin JD, Gordon MK, et al. Regulation of TREM expression in hepatic macrophages and endothelial cells during acute endotoxemia. Exp Mol Path 2008;84:145-155.

32. Kaplan AP. Chemokines, chemokine receptors and allergy. Int Arch Allergy Immunol 2001;124:423-431.

33. Gu Y, Lewis DF, Groome LJ, et al. Elevated maternal il-16 levels, enhanced il-16 expressions in endothelium and leukocytes, and increased il-16 production by placental trophoblasts in women with preeclampsia. J Immunol 2008;181:4418-4422.

34. Pap $E$, Pallinger $E$, Pasztoi $M$, et al. Highlights of a new type of intercellular communication: microvesicle-based information transfer. Inflamm Res 2009;58:1-8.

35. Cocucci E, Racchetti G, Meldolesi J. Shedding microvesicles: artefacts no more. Trends Cell Biol 2009;19:43-51.

36. Soleti R, Benameur T, Porro C, et al. Microparticles harboring sonic hedgehog promote angiogenesis through the upregulation of adhesion proteins and proangiogenic factors. Carcinogenesis 2009;30:580-588.

37. Lynch SF, Ludlam CA. Plasma microparticles and vascular disorders. Br J Haematol 2007;137:36-48.

38. Lechner D, Weltermann A. Circulating tissue factor-exposing microparticles. Thromb Res 2008;122:S47-S54.

39. Mostefai HA, Andriantsitohaina R, Martinez MC. Plasma membrane microparticles in angiogenesis: role in ischemic diseases and in cancer. Physiol Res 2008;57:311-320.

40. Aharon A, Brenner B. Microparticles, thrombosis and cancer. Best Pract Res Clin Haematol 2009;22:61-69.

41. Kim HK, Song KS, Chung JH, et al. Platelet microparticles induce angiogenesis in vitro. Br J Haematol 2004;124:376-384.

42. Leroyer AS, Rautou PE, Silvestre JS, et al. CD40 ligand+ microparticles from human atherosclerotic plaques stimulate endothelia proliferation and angiogenesis a potential mechanism for intraplaque neovascularization. J Am Coll Cardiol 2008;52: 1302-1311.

43. Mezentsev A, Merks RM, O'Riordan E, et al. Endothelial microparticles affect angiogenesis in vitro: role of oxidative stress. Am J Physiol Heart Circ Physiol 2005;289:H1106-H1114.

44. Yang C, Mwaikambo BR, Zhu T, et al. Lymphocytic microparticles inhibit angiogenesis by stimulating oxidative stress and negatively regulating VEGF-induced pathways. Am J Physiol Regul Integr Comp Physiol 2008;294:R467-R476.
45. Janowska-Wieczorek A, Wysoczynski M, Kijowski J, et al. Microvesicles derived from activated platelets induce metastasis and angiogenesis in lung cancer. Int J Cancer 2005;113:752-760.

46. Kim CW, Lee HM, Lee $\mathrm{TH}$, et al. Extracellular membrane vesicles from tumor cells promote angiogenesis via sphingomyelin. Cancer Res 2002;62:6312-6317.

47. Millimaggi D, Mari M, D'Ascenzo S, et al. Tumor vesicle-associated CD147 modulates the angiogenic capability of endothelial cells. Neoplasia 2007;9:349-357.

48. Taraboletti G, D'Ascenzo S, Giusti I, et al. Bioavailability of VEGF in tumor-shed vesicles depends on vesicle burst induced by acidic $\mathrm{pH}$. Neoplasia 2006;8:96-103.

49. Skog J, Wurdinger T, van Rijn S, et al. Glioblastoma microvesicles transport RNA and proteins that promote tumour growth and provide diagnostic biomarkers. Nat Cell Biol 2008;10:1470-1476.

50. Al-Nedawi K, Meehan B, Micallef J, et al. Intercellular transfer of the oncogenic receptor EGFRvIll by microvesicles derived from tumour cells. Nat Cell Biol 2008;10:619-624.

51. Gesierich S, Berezovskiy I, Ryschich E, et al. Systemic induction of the angiogenesis switch by the tetraspanin D6.1A/CO-029. Cancer Res 2006;66:7083-7094.

52. Hanahan D, Folkman J. Patterns and emerging mechanisms of the angiogenic switch during tumorigenesis. Cell 1996;86:353-364.

53. Valenti $R$, Huber $V$, lero $M$, et al. Tumor-released microvesicles as vehicles of immunosuppression. Cancer Res 2007;67:2912-2915.

54. Bin KJ. Three-dimensional tissue culture models in cancer biology. Semin Cancer Biol 2005;15:365-377.

55. Valster A, Tran NL, Nakada M, et al. Cell migration and invasion assays. Methods 2005;37:208-215.

56. Yamada KM, Cukierman E. Modeling tissue morphogenesis and cancer in 3D. Cell 2007;130:601-610.

57. Zhou Q, Kiosses WB, Liu J, et al. Tumor endothelial cell tube formation model for determining anti-angiogenic activity of a tRNA synthetase cytokine. Methods 2008;44:190-195.

58. Sun X-T, Zhang M-Y, Shu C, et al. Differential gene expression during capillary morphogenesis in a microcarrier-based three-dimensional in vitro model of angiogenesis with focus on chemokines and chemokine receptors. World J Gastroenterol 2005;11:2283-2290.

59. Taylor DD, Gercel-Taylor C. MicroRNA signatures of tumor-derived exosomes as diagnositic biomarkers of ovarian cancer. Gynecol Onc 2008;110:13-21. 